\title{
A computational model for the excitatory network of the C2 column of barrel cortex Christian Tomm*1, Sandrine Lefort ${ }^{2}$, Juan-Carlos Floyd Sarria ${ }^{3}$ and Carl CH Petersen ${ }^{2}$
}

\author{
Address: ${ }^{1}$ Laboratory of computational Neuroscience, EPFL, Lausanne, 1015 Switzerland, ${ }^{2}$ Laboratory of Sensory Processing, EPFL, Lausanne, \\ 1015 Switzerland and ${ }^{3}$ Bioimaging and Optics, EPFL, Lausanne, 1015 Switzerland \\ Email: Christian Tomm* - christian.tomm@epfl.ch \\ * Corresponding author
}

from Eighteenth Annual Computational Neuroscience Meeting: CNS*2009

Berlin, Germany. 18-23 July 2009

Published: 13 July 2009

BMC Neuroscience 2009, I0(Suppl I):PI64 doi:I0.II86/I47|-2202-I0-SI-PI64

This abstract is available from: http://www.biomedcentral.com/I47I-2202/I0/SI/PI64

(C) 2009 Tomm et al; licensee BioMed Central Ltd.

Many animals, especially rodents, use whiskers as an important sensory input. One of the multiple interesting properties of the whisker sensory pathway is its somatotopic mapping. Every single whisker has a local representation in the primary sensory cortex. In this barrel cortex, each whisker is represented by a single column of cortex. The excitatory connections of the $\mathrm{C} 2$ barrel cortex column were researched thoroughly in [1].

Using these data, a model was built to mirror the measured pair to pair connectivity. This model consists of integrate and fire neurons and uses the cell counts found in the experiments. Only taking into account the excitatory population, this amounts to around 6000 neurons in the network. The neuronal properties such as threshold, resting potential, EPSP shape, etc. are fitted to the found experimental values. Through simulations we show how the distribution of synaptic weights can influence the behaviour of the network.

\section{References}

I. Lefort S, Tomm C, Floyd Sarria JC, Petersen CC: The excitatory neuronal network of the $\mathrm{C} 2$ barrel column in mouse primary somatosensory cortex. Neuron 2009, 6I:30I-316. 